\title{
Faraday's Iron Ring Experiment (1831) Explained with Gill's Electronic Theory of Magnetism (1964)
}

\author{
Avtar Singh Gill \\ avtargillmd@aol.com
}

Received Date: May 14, 2017

Accepted Date: June 28, 2017

Published Date: July 05, 2017

Abstract: Gill's electronic theory of magnetism (1964) was put forward by the author to explain a change in configuration of the atoms which then start to behave like magnets. The author does not agree with the pre-existing dipole theory of Maxwell (1873).

By applying Gill's electronic theory of magnetism (1964) to Faraday's (1831) iron ring experiment, the unexpected result obtained by Michael Faraday in 1831 will be explained.

Using Coulomb's law, dot-product calculations and equations have been developed by the author versus the cross-product Lorentz equations (1893).

Magnetism and the Tesla unit will be addressed and it will be shown with the help of Coulomb's law that if we have $\boldsymbol{n}=\frac{1}{\sqrt{\boldsymbol{k}} \cdot \boldsymbol{e}}=(\sqrt{\boldsymbol{k}} \cdot \boldsymbol{e})^{-1}$ non-moving inner electrons at the north magnetic pole and have at a distance of one meter the same number of exposed protons as the south magnetic pole, then we will experience a magnetic force of one Tesla between the two magnetic poles.

The issue of asymmetry between magnetic force and electrical force pointed out by A. Einstein in 1905 and Richard Feynman in 1943 is resolved by applying Gill's electronic theory of magnetism (1964) instead of Maxwell's dipole theory of magnetism (1873).

Introduction: This article has been written to explain the results obtained by Michael Faraday in his 1831 iron ring experiment with the application of Gill's electronic theory of magnetism (1964). The ability to do the same also lends support to Gill's electronic theory of magnetism. It will be shown with line diagrams and a simple experiment that the magnetic force is a combination of positive and negative forces from the protons and electrons of a re-configured magnetized atom. The author will go on to derive dot product calculations and equations after having failed to reconcile with the cross-product Lorentz formula of 1893. The derived equations are applied to define and calculate a Tesla unit. Maxwell's dipole theory of magnetism (1873) causes the asymmetry issue and it will be shown that Gill's electronic theory of magnetism resolves the asymmetry issue.

Conclusion: Application of Gill's electronic theory of magnetism (1964) shows how a centrifugal force is created and manifests and travels on the surface from the northpole to the south pole of a magnet during magnetization and in the opposite direction during demagnetization and manifests only at the ends of a magnet otherwise.

It will be shown that both during magnetism and electricity, the interaction is between positive and negative forces of an atom. During electrical current we have the free valence electrons flowing in the conducting coil. During magnetism, the atom undergoes a change in configuration between its electrons and protons. Dot product equations suffice.

Method: Gill's electronic theory of magnetism (1964) will be summarized followed by a simple experiment to show that the fundamental magnetic force is a combination of proton based positive and electron based negative forces. Next, a simple thought experiment involving Faraday's iron ring experiment (1831) to show how opposite induction works. Next will be the actual Faraday's iron ring experiment (1831) and how the application of Gill's electronic theory of magnetism (1964) helps in explaining the magnetic induction followed by the electrical induction. This will be followed by dot product calculations developed by the author instead of the cross-product equations of Lorentz (1893). Finally, the asymmetry issue will be addressed and resolved if you apply Gill's electronic theory of magnetism instead of Maxwell's dipole dependant theory.

\section{Gill’'s Electronic Theory of Magnetism (1964)}

This is based on the structure of the atom and explains how the positively charged protons and the negatively charged electrons are responsible for both magnetism and electrical forces. 
In the diagrams that follow in this article, we are using a simplified version of the structure of an atom with a large black proton and small red electrons.

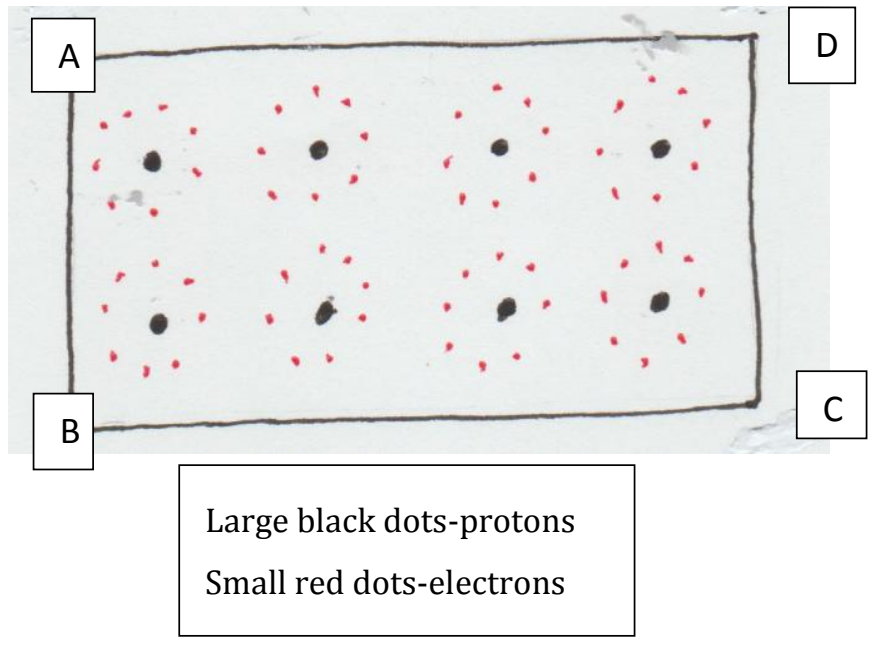

Figure1 (a): above shows neutral iron atoms in an un-magnetized state.

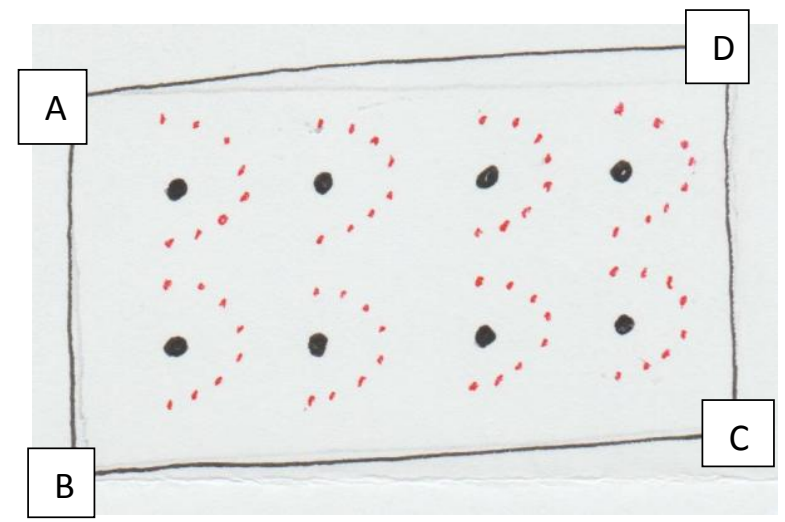

Figure1 (b): above shows the change in position of the inner electrons on magnetization.

Gill's electronic theory of magnetism (1964) shows the neutral iron atoms in Fig 1a are magnetized in Figure $1 \mathrm{~b}$ and $\mathrm{CD}$ has become the negative magnetic pole or north pole with a torqued non-moving charge - $\boldsymbol{n} \boldsymbol{e}$ of the magnet and $\mathrm{AB}$ has become the positive magnetic pole or the south pole of the magnet with an oppositely torqued non-moving charge $+\boldsymbol{n} \boldsymbol{e}$ where $\boldsymbol{n}$ is the number of exposed inner electrons at one end and equals the number of exposed protons at the other end. The neutral atoms in Fig 1a have become magnetized atoms in Fig $1 b$ by undergoing a change in configuration and each atom also has developed an opposing torque between its own electrons and protons to give the magnetized atoms a corkscrew effect.
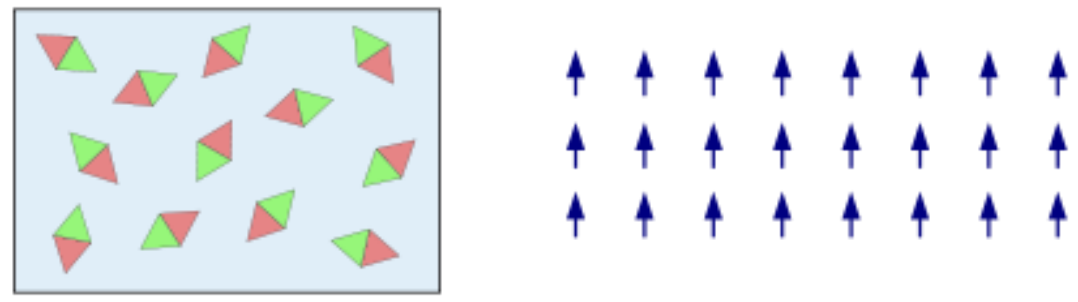

Figure1 (c): ferromagnetic metals consisting of tiny dipole magnets

Maxwell's Theory of Electro-Magnetism (1873) as shown in Figure 1c above describes ferromagnetic metals consisting of tiny dipole magnets (every north pole is yoked to a south pole) which straighten out during magnetization into north to south direction and in a non-magnetic state they are a jumbled lattice which does not manifest any free North-pole and South-pole which does happen when the same is magnetized as explained in the figure to the right. 
Figure 1c leaves us with the erroneous notion that the magnetic force is a single fundamental force of magnetism. However, the line diagrams presented in Figure 1b show that the magnetic force comprises of two forces emanating from the proton dependant south magnetic pole and the electron dependant north magnetic pole of reconfigured magnetized atoms.

EXPERIMENT

A physicist showed me the following experiment in 1965. On a wooden table, spread some coarse iron filings and in the middle of the iron filings, place a magnet.

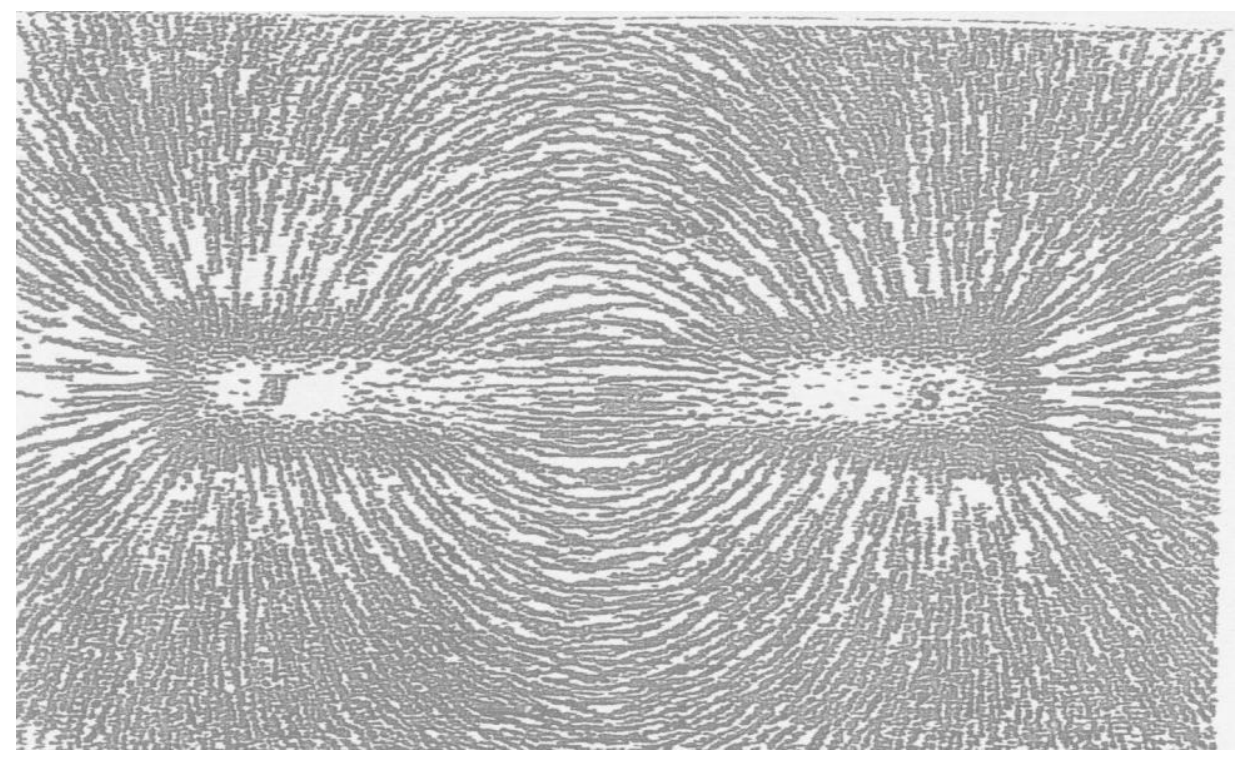

Figure2 (a): shows a bar magnet with iron filings arranged along magnetic field lines

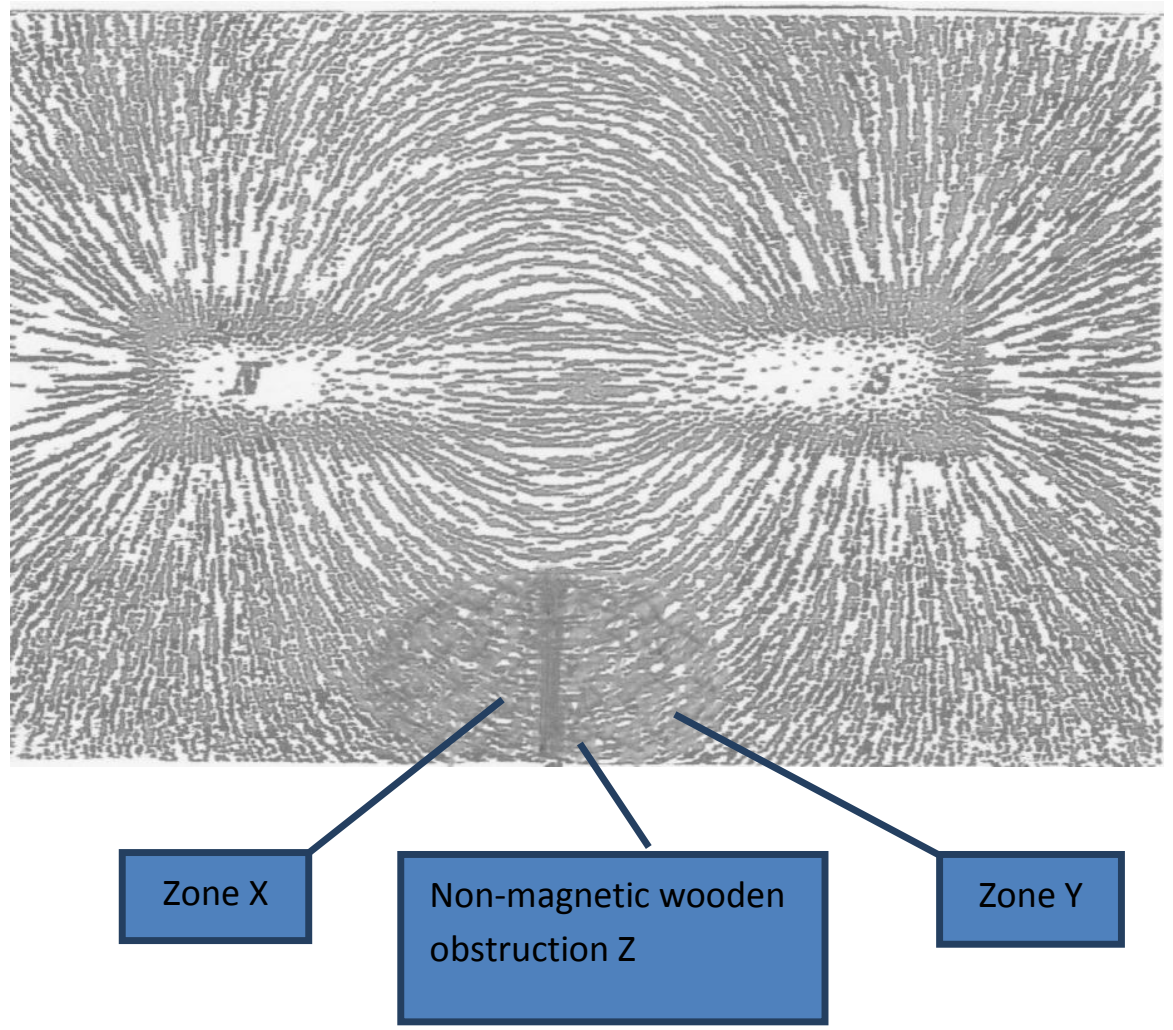

Figure2 (b): a wooden non-magnetic obstruction $Z$ is placed on one side on the iron filings

In Fig 2 b, a wooden non-magnetic obstruction $\mathrm{Z}$ is placed on one side on the iron filings. The iron filings crumple on both sides of $\mathrm{Z}$ in zones $\mathrm{X}$ and $\mathrm{Y}$. If the magnetic force was a single force, the iron filings should have crumpled in Zone X or Zone Y only. 
Thus, the magnetic force is combination of the positive and negative forces from the two poles of a magnet seen both diagrammatically and experimentally.

\section{FARADAY'S IRON RING EXPERIMENT (1831)}

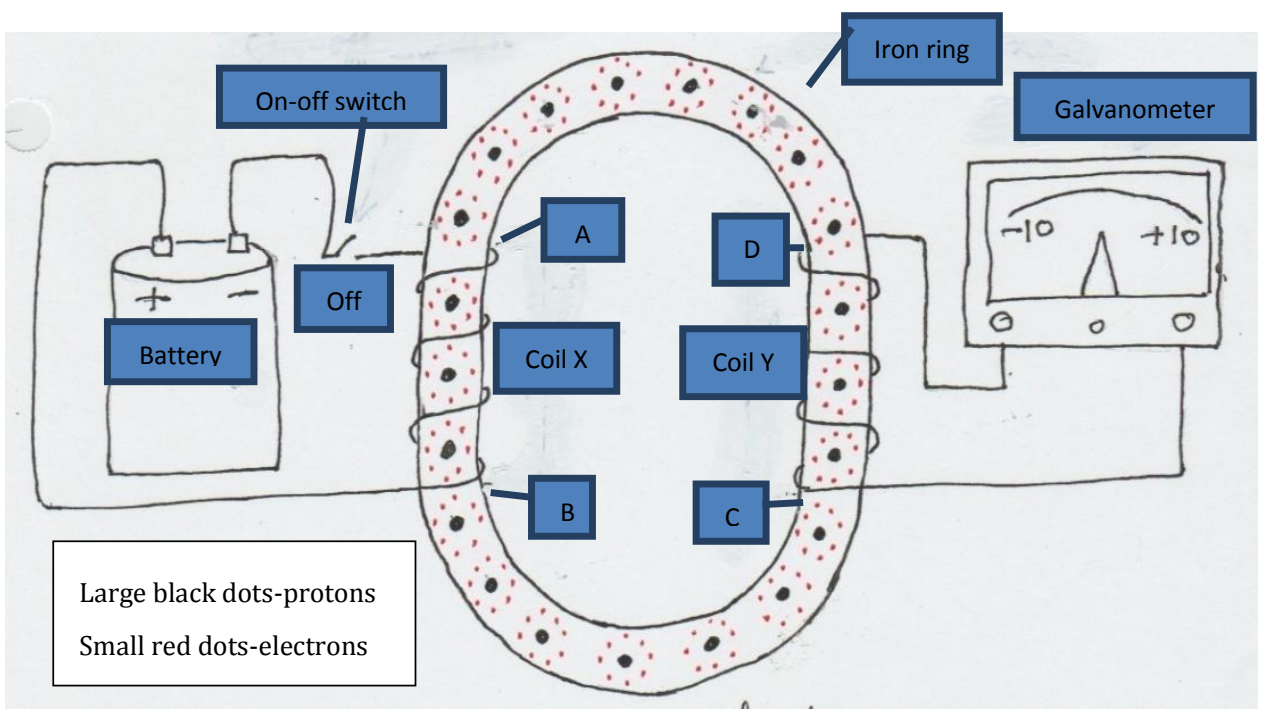

Figure 3

Faraday's experiment to try to induce an electric current from a magnetic field has a battery on the left, an iron ring in the centre and a galvanometer on the right. The left coil $\mathrm{X}$ is around the iron ring from point $\mathrm{A}$ to point $\mathrm{B}$ and it is connected to a battery. The right coil $\mathrm{Y}$ is around the iron ring from point $\mathrm{C}$ to point $\mathrm{D}$ and is connected to a galvanometer and no battery is connected to coil Y. In the iron ring, we have arranged a row of neutral atoms each with a central proton mass (large black dot) surrounded by electrons (small red dots).

Faraday saw the galvanometer in circuit $\mathrm{Y}$ deflect at the moment he closed the switch in circuit X and an opposite galvanometer deflection was seen when the battery was switched off.

\section{A thought experiment to explain the opposite induction in coil Y}

The iron ring has been made oblong as it will help with the calculations later, although nothing changes.

\section{SWITCH TURNED ON WITH ONE COMPLETE COIL AROUND THE ENTIRE IRON CORE RING}

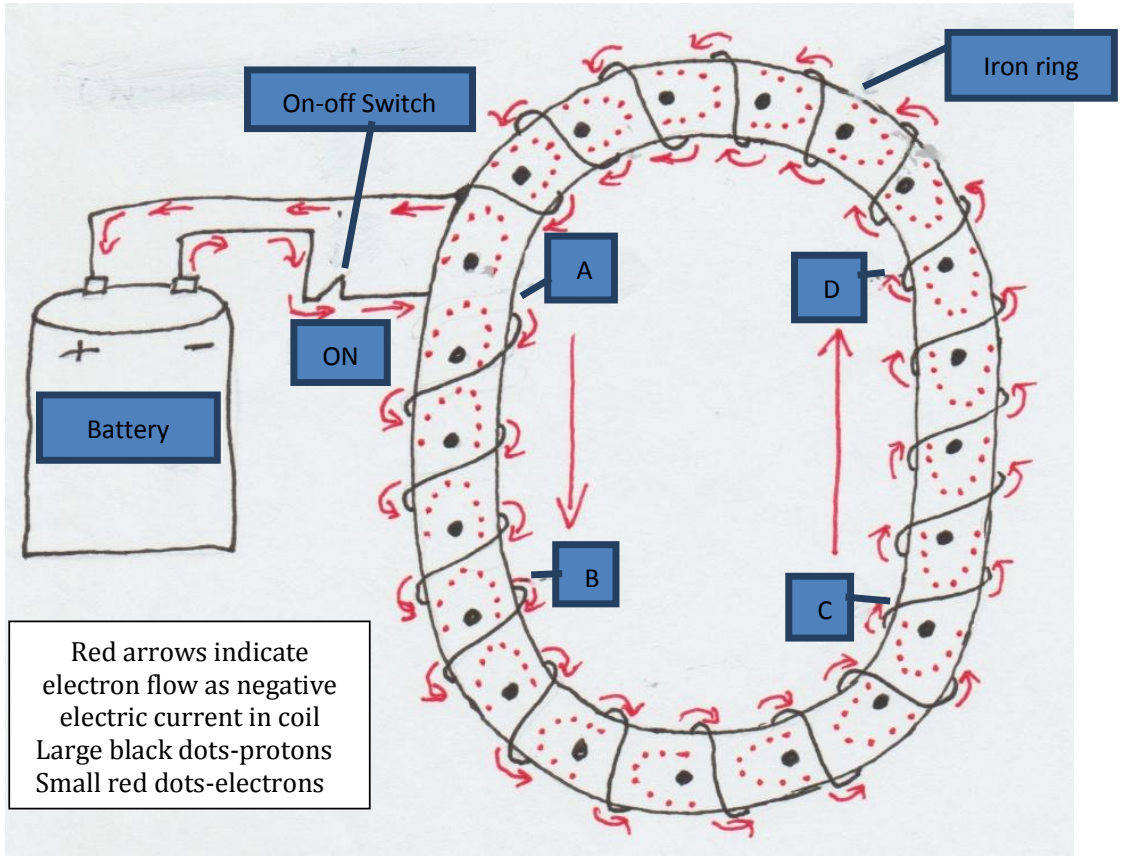

Figure 4 
Although the negative electric current flow in the coil is clockwise around the iron ring from $A$ to $B$, it becomes counter-clockwise from $C$ to $D$ as the direction of the ring has changed by 180 degrees.

The overall electron based negative electric current is flowing clockwise and the protons of each atom are overall pulled/attracted clockwise and the inner electrons of each atom in the iron ring are pushed/repelled in the counter-clockwise direction causing a torsionin the atom due to the circular flow of the electrons in the coil. Also, the protons in the atom in iron ring will be pulled from $A$ to $B$ as the electrons are flowing from $A$ to $B$ and the electrons of the atom will be repelled from $B$ to $A$ by this electron flow. Each magnetized atom now has exposed inner electrons towards $A$ and exposed protons towards $B$.

This magnetization of the iron ring atoms starts from point $A$ where the initial electric current reaches the iron ring and travels with the negative electric current like a chain all the way around according to Gill's electronic theory of magnetism(1964).

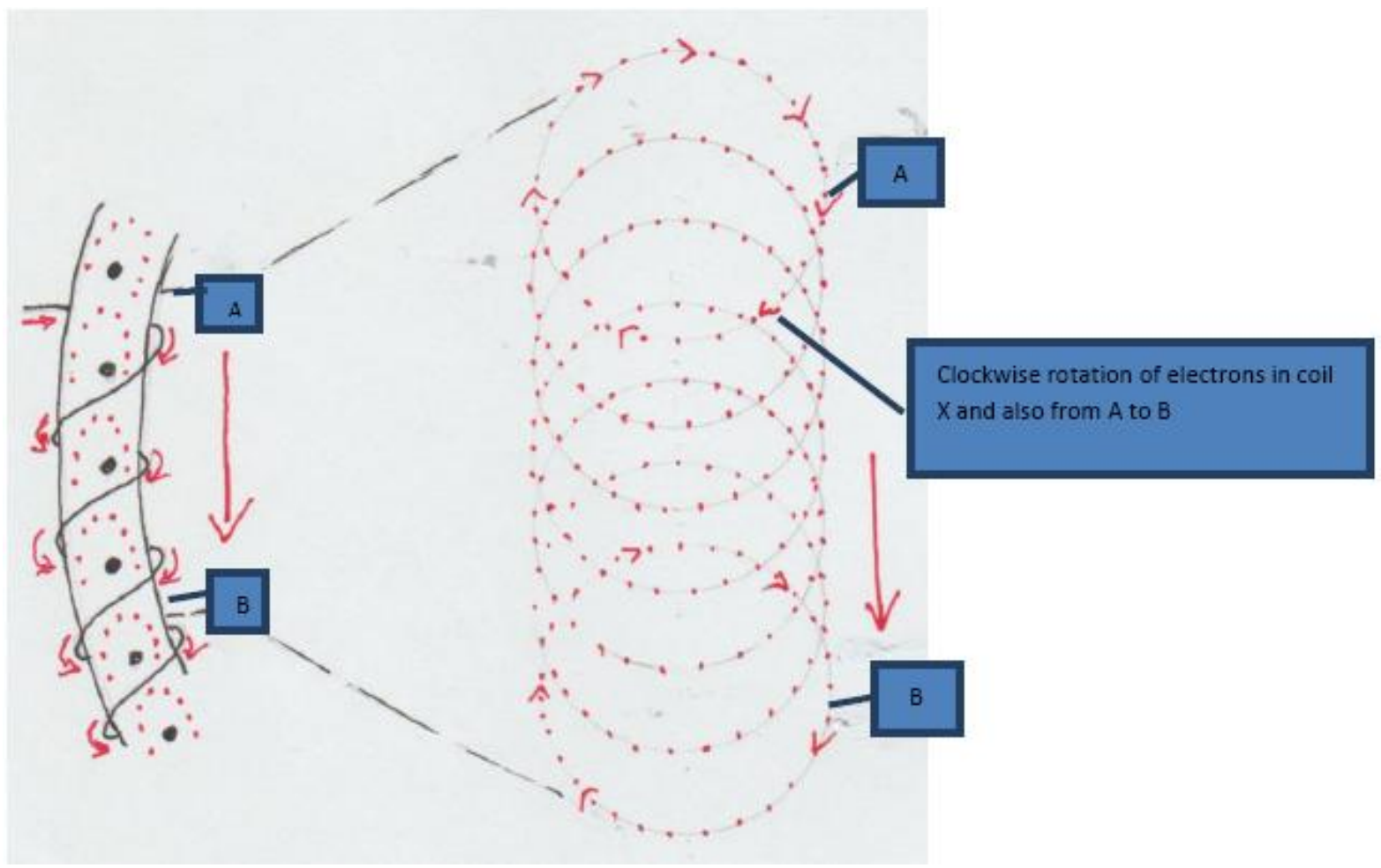

Figure 5

In above Figure 5, the electrons in coil $\mathrm{X}$ which are causing the negative electric current are flowingfrom point $\mathrm{A}$ to point $\mathrm{B}$ and these electrons in coil $\mathrm{X}$ are flowing1-in a circular clockwise direction as a centripetal force around the iron ring and 2-this centripetal force moves longitudinally in the coil from $A \rightarrow B$ in a tubular manner.

Whereas the circular flowing electrons in the coil X will cause a centripetal force on the atoms of the iron ring causing anegative torque $(-\boldsymbol{\tau})$ on the protons of an iron ring atom and a positive torque $(+\boldsymbol{\tau})$ on the electrons of the same atom, it is the tubular flow of electrons from $A \rightarrow B$ which will repel the inner electrons of that atom in the iron core upwards to cause the north or negative magnetic pole andpull the protons of each atom in the iron ring downwards to cause the south or positive magnetic pole during magnetization of that atom in the iron ring.

Even if the negative electric current is only from $A \rightarrow B$, beyond the point $B$, each of these magnetised atoms will cause a similar change in the next atom and the next in the iron core and the iron core atoms will continue to be magnetized sequentially from $\mathrm{A} \rightarrow \mathrm{B} \rightarrow \mathrm{C} \rightarrow \mathrm{D} \rightarrow \mathrm{A}$ with the application of Gill's electronic theory of magnetism. 


\section{FORCES ACTING ON PROTONS AND ELECTRONS OF ATOMS IN IRON RING BY ELECTRIC COIL}

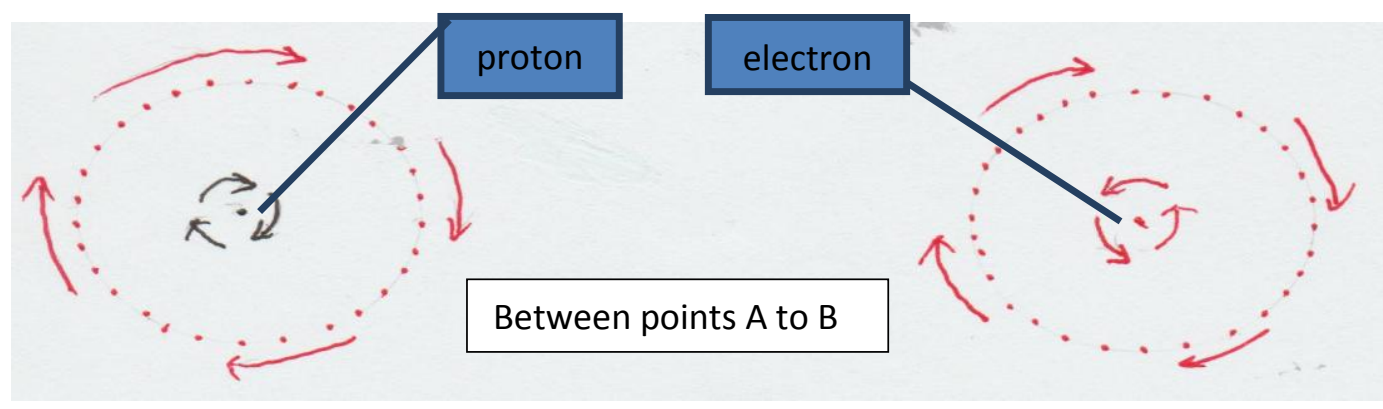

Figure 6(a)

In above Figure $6 \mathrm{a}$, as the electrons in conducting coil are rotating clockwise between $\mathrm{A}$ and $\mathrm{B}$, they cause a centripetal force of attraction towards proton of an iron core atom and torque it clockwiseas a negative torque $(-\boldsymbol{\tau})$. They will also cause a centripetal force of repulsion on an adjacent inner electron of the atom in the iron core and torque it anticlockwise as a positive torque $(+\boldsymbol{\tau})$.

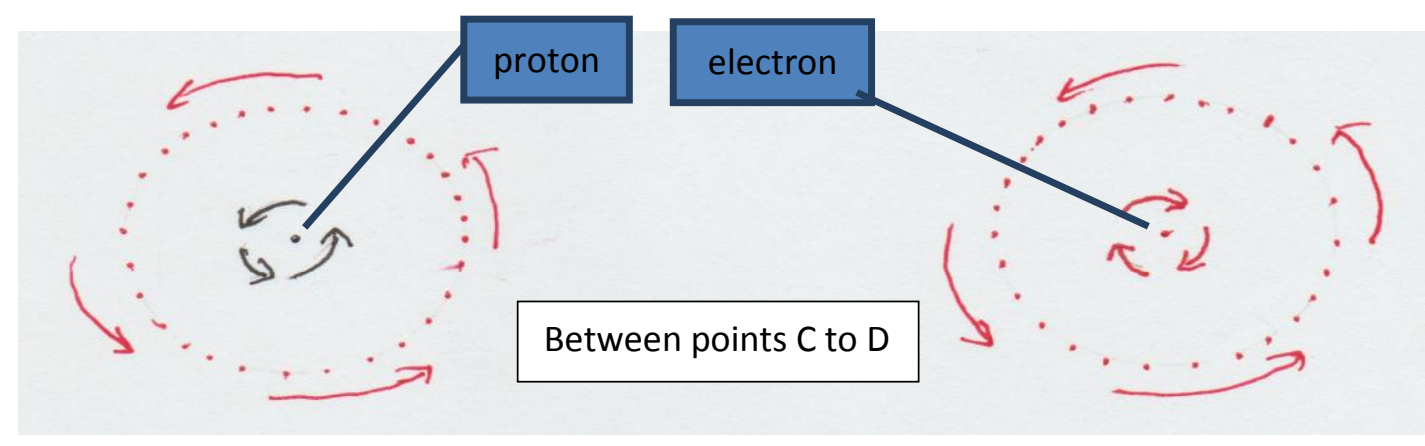

Figure 6(b)

In Figure $6 \mathrm{~b}$, between points $\mathrm{C}$ and $\mathrm{D}$, as the electrons in the conducting coil are flowing anti-clockwise owin to a 180 degree change in direction, the protons will be torqued anti-clockwise as a positive torque $(+\boldsymbol{\tau})$ and the electrons of the iron core atoms between $\mathrm{C}$ and $\mathrm{D}$ will be torqued clockwise $(-\boldsymbol{\tau})$. This torque is opposite to that between points A to B.

(Magnetized) atom in iron core between $A \rightarrow B$ :

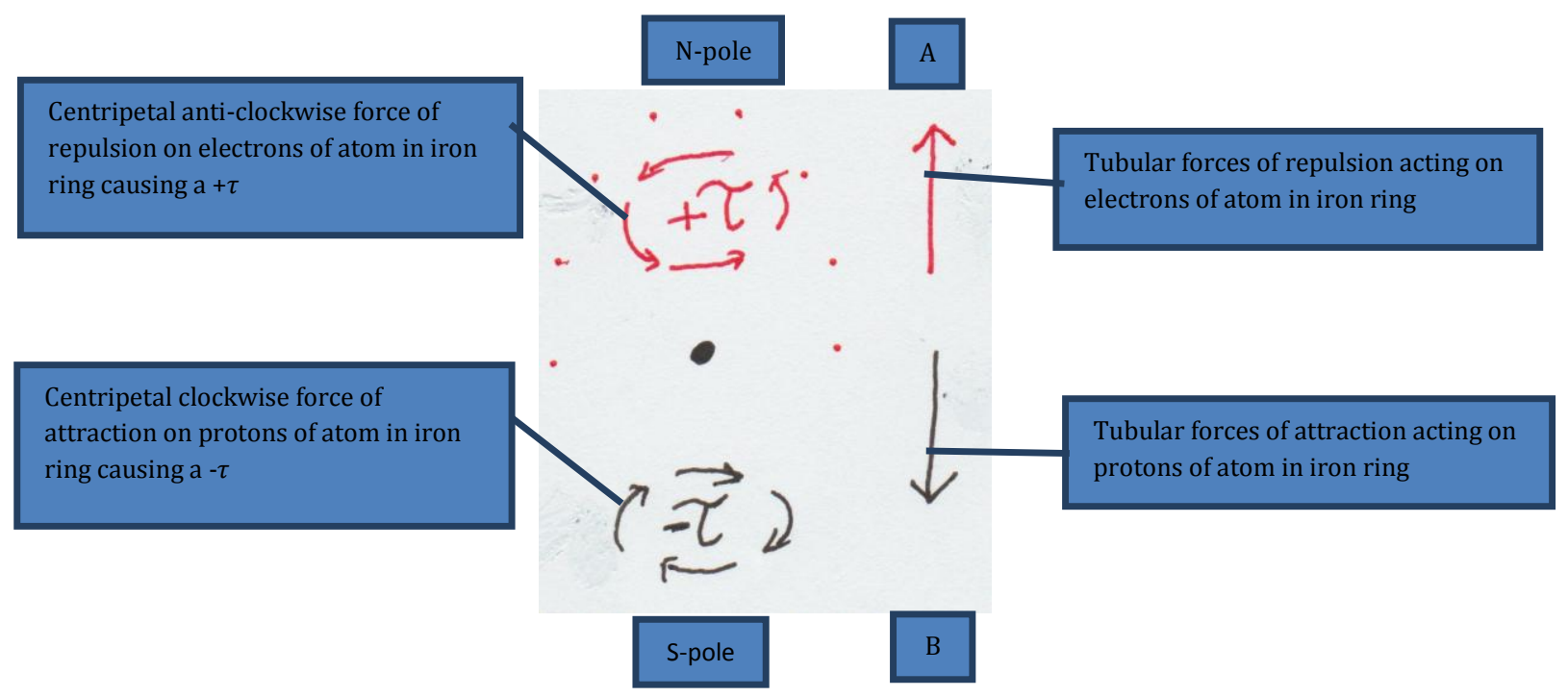

Figure7: Magnetized atom in iron ring between $A$ and $B$ 
In Figure 7, between points $A$ and $B$, the flowing electrons in coil $\mathrm{X}$ exert a force on the protons and the electrons of the atoms in the iron ring:

1- torque the protons in each atom in the clockwise direction $(-\tau)$ due to attraction.

2-attractthe protons of each atom in the iron ring downwards in the direction $A \rightarrow B$. Also, these flowing electrons in coil X from $A \rightarrow B$

3 -torque the inner electrons of iron ring atom in the anti-clockwise direction $(+\boldsymbol{\tau})$ due to repulsion.

4-repel the electrons in each atom in the iron ring upwards in the direction $B \rightarrow A$.

The longitudinally moving centripetal force exerted by the circular electron flow in the conducting coil causes positive torque $(+\boldsymbol{\tau})$ on the electrons and negative torque $(-\boldsymbol{\tau})$ on protons of the iron ring atoms and the tubular flow of electrons starting from point $A$ and movingfrom $A \rightarrow B$ causes the atoms of the iron core to be magnetized sequentially from $A \rightarrow B$.The torqued negative electron dependant north magnetic pole and the oppositely torqued positive proton dependant south magnetic pole of a magnetized atom result from the circular and tubular flow of electrons in coil X from $A \rightarrow B$.

\section{(Magnetized) atom in iron ring from $C \rightarrow D$}

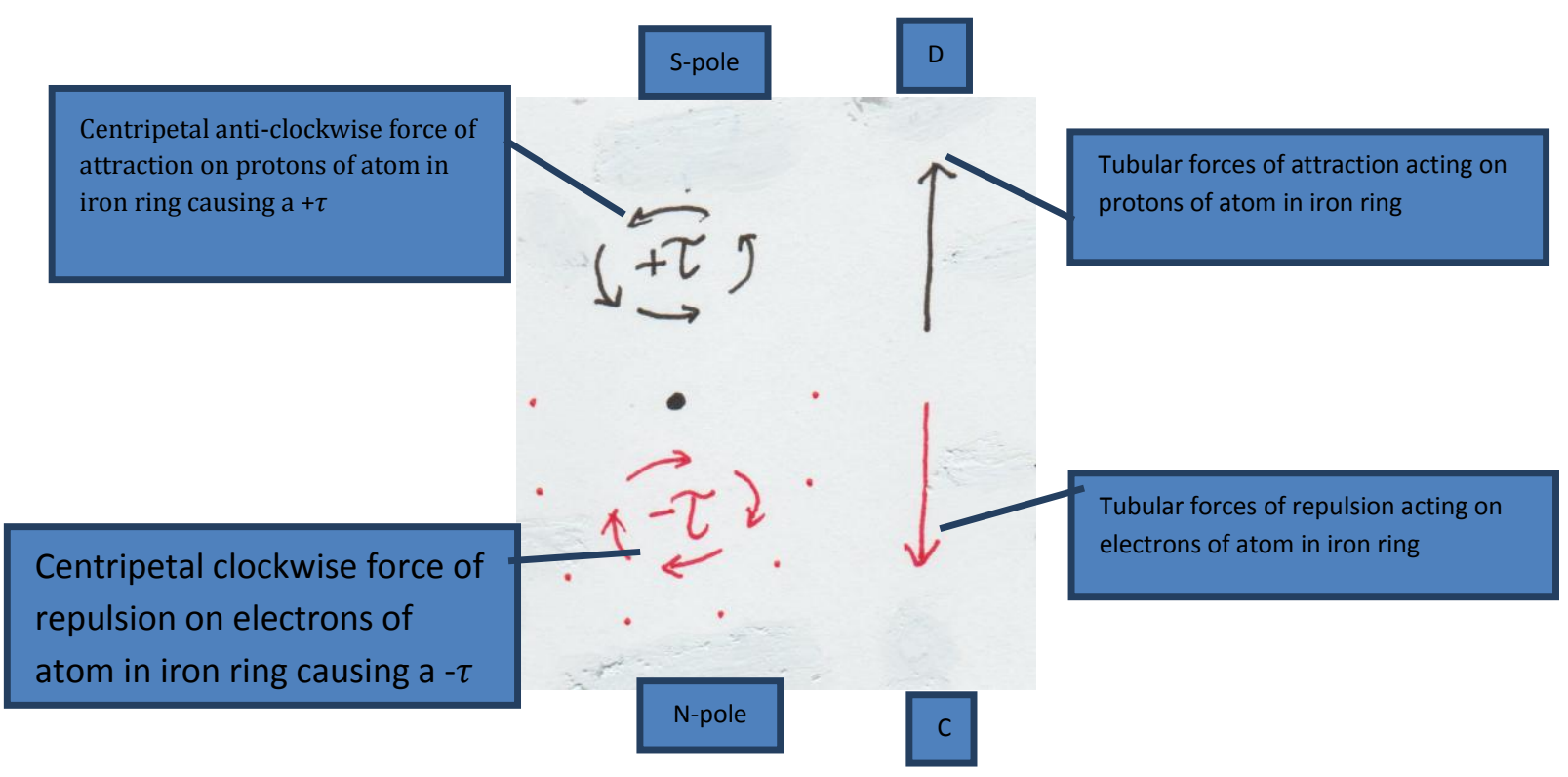

Figure8: Magnetized atom in iron ring between $C$ and $D$

In Figure 8, we are looking at a (magnetized) atom in the iron core ring between points $\mathrm{C}$ and $\mathrm{D}$. The direction of the coil has changed 180 degrees and although the electric current electrons are moving from point $\mathrm{C}$ towards point $\mathrm{D}$ in a tubular manner, the circular flow of negative electric current has become anticlockwise owing to the 180 degree change in direction. The atoms in the iron ring obey Ampere's right hand rule (discussed later) and have the electron dependant north or negative magnetic pole towards $\mathrm{C}$ and the proton dependant south or positive magnetic pole towards point D. As the electrons in this part of the coil are flowing counter-clockwise, the torque on the proton dependant south magnetic pole of the atom is $(+\boldsymbol{\tau})$ and the torque on the electron dependant north magnetic pole of the atom is $(-\boldsymbol{\tau})$.

It is this 180 degree change of direction from $C \rightarrow$ Das compared to $A \rightarrow B$ which is responsible for inducing an electric current in the opposite direction as seen by Faraday in 1831.

Even if a part of the iron ring is subjected to an electric current by coil $X$, the entire iron core ring will be magnetized sequentially as each magnetized atom will cause a similar change in the next atom and the chain continues all the way around as explained by Gill's electronic theory of magnetism (1964). 


\section{SWITCH TURNED ON IN FARADAY'S 1831 EXPERIMENT}

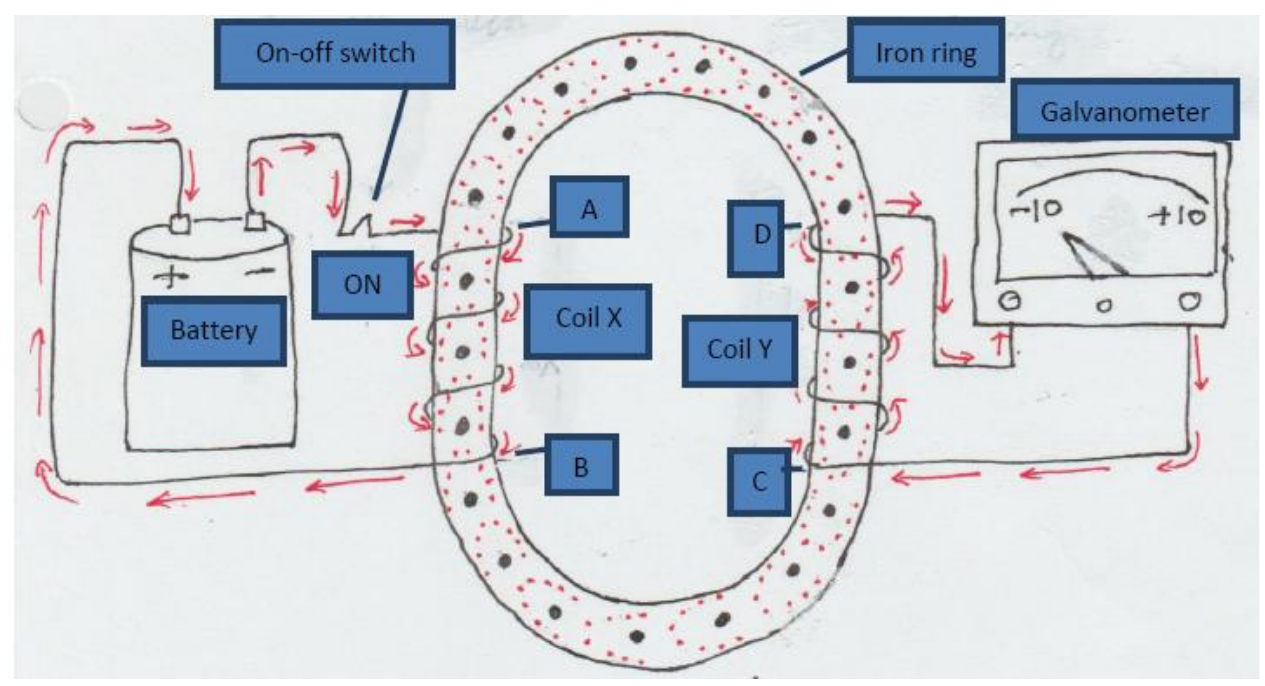

Red arrows indicate direction of flow of electrons with negative electricity in conducting coils

Large black dots-protons

Small red dots-electrons

Figure 9

In Figure 9, as soon as the switch of the battery is turned on, the outer free negatively charged valence electrons start flowing as an electric current in the conducting coil X starting from point $A$ in a clockwise direction and from above downwards in a tubular manner from $A \rightarrow B$. The atoms in the iron ring will be magnetized sequentially along with the flow of negative electric current from $A \rightarrow B$ and the inner electrons of each iron ring atom will be repelled towards point $A$ and undergo a positive torque $(+\tau)$ and the protons of the same atom will be attracted towards point $B$ and undergo a negative torque $(-\tau)$.

Each magnetized atom in the iron ring will subject the next neutral atom in the iron ring to get magnetized like itself by attracting the inner electrons of that atom and repelling the protons of the second atom and also transmitting a similar torque $(\boldsymbol{\tau})$ to the protons and electrons of the next atom like itself and the chain continues until the entire ring is magnetized. This process of magnetization of the atoms in the iron ring travels in the same direction as the flow of electric current in coil $\mathrm{X}$ and continues sequentially all the way from $A \rightarrow B \rightarrow C \rightarrow D \rightarrow A$.

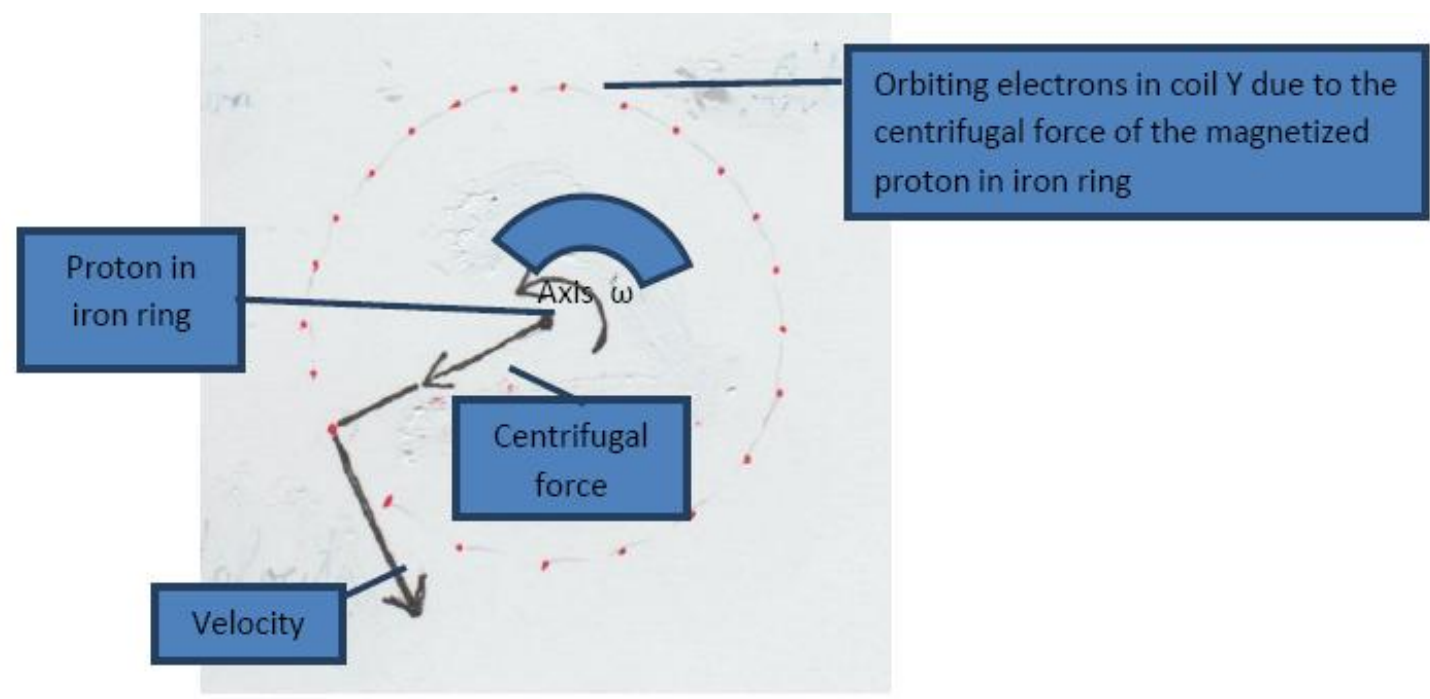

Figure 10(a) 
From point $\mathrm{C}$ to point $\mathrm{D}$, the atoms in the iron core are being magnetized sequentially from $C \rightarrow D$ and are also undergoing a torque which exerts a travelling centrifugal force from $C \rightarrow D$ which acts on the free valence electrons in coil Y causing it to manifest as an electric current only during magnetization. Owing to a 180 degree change in the direction, the travelling centrifugal force from $C \rightarrow D$ is because of the inner electrons of the atoms in iron ring developing a negative torque $(-\boldsymbol{\tau})$ and the protons of the same atom develop a positive torque $(+\boldsymbol{\tau})$ which is opposite to that between $A \rightarrow B$.

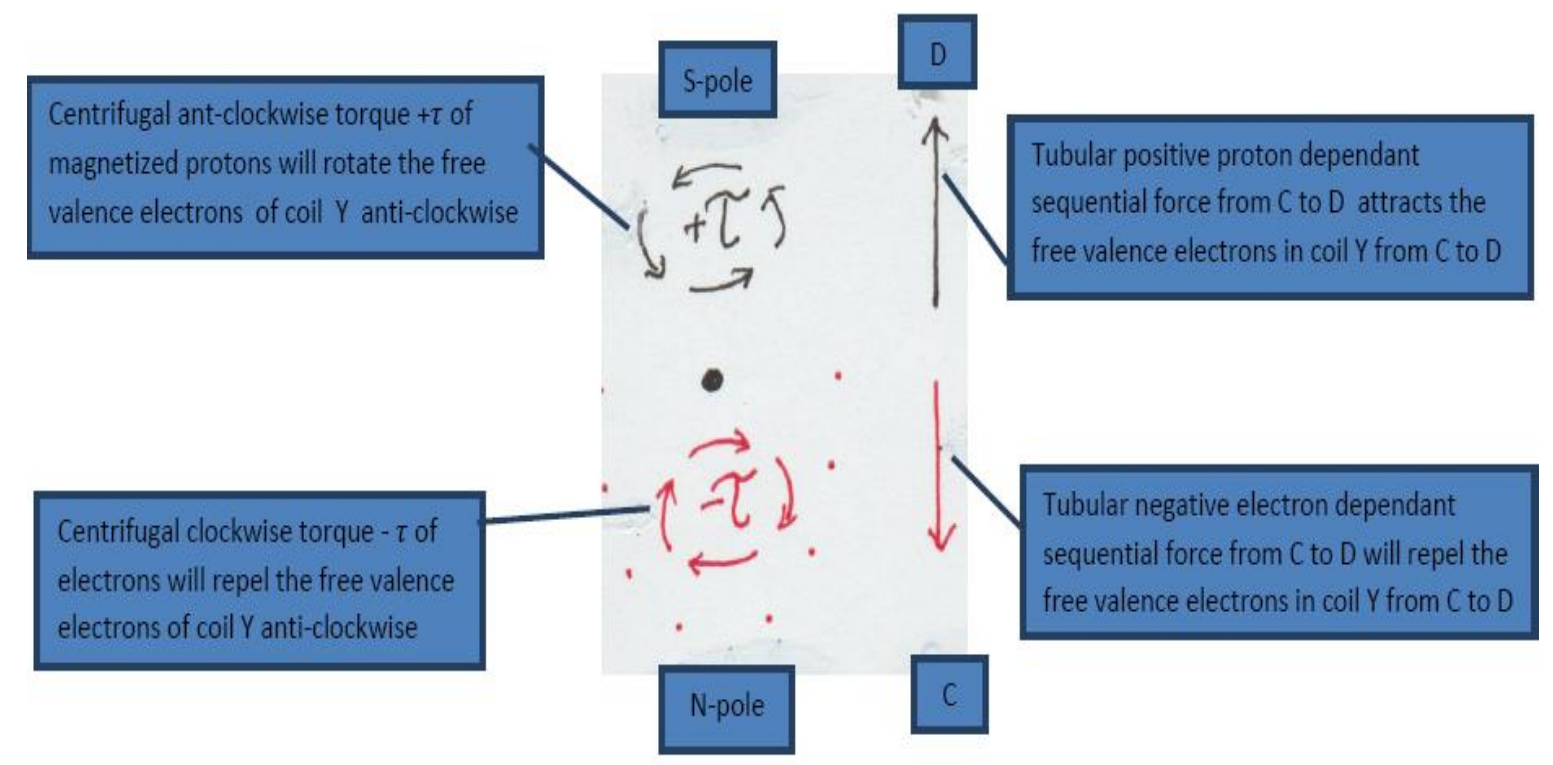

Figure 10(b). Magnetized atom of iron ring between $C$ and $D$ inside coil $Y$

In Figure $10 \mathrm{~b}$ we see a magnetized atom in the iron core ring in the section $C \rightarrow D$ where the conducting coil Y has been placed connected only to a galvanometer and there is no battery. As the magnetization is spreading from $C \rightarrow D$, the direction of the magnetized atoms is opposite to those in section $A \rightarrow B$ and they have their positive south magnetic pole pointed towards $\mathrm{D}$ and their negative north magnetic pole pointed towards C. Also, the magnetized atoms from $C \rightarrow D$ are torqued in the opposite direction as compared to the magnetized atoms from $A \rightarrow B$ owing to a 180 degree change in direction.

Just when these atoms in the iron core are being magnetized, each of these atoms during magnetization will exert a centrifugal circular force (which also propagates longitudinally in a chain-like manner owing to a sequential magnetization from $C \rightarrow D$ on the free valence electrons of a neutral conducting wire $\mathrm{Y}$ and cause the free valence electrons in coil $\mathrm{Y}$ to flow resulting in an induced current. The positive anticlockwise torque of the suddenly magnetized exposed protons $(+\boldsymbol{\tau})$ whichcause a centrifugal force of attraction causing the free valence electrons in the coil $Y$ to be rotated anti-clockwise and the longitudinal chain-like force from $C \rightarrow D$ will attract these free valence electrons in coil Y upwards from $C \rightarrow D$.This is in the opposite direction as compared to electron flow in coil $\mathrm{X}$ and we see the induction of an opposite induced electric current in coil Y seen on the galvanometer deflection only while the iron ring is being magnetized.

\section{STEADY STATE}

When the iron ring has reached a temporary steady state of magnetization due to a steady direct electric current from the battery, there is no further magnetization and thus no more travelling centrifugal force from $C \rightarrow D$ due to the protons and electrons of the magnetized atoms of the iron ring. Hence, there is no more electrical current in coil Y during the steady state.

It be noted that the travelling centrifugal force manifests on the surface of iron ring only at magnetization or demagnetization. Ampere in 1825 mentioned a travelling force on the surface of a magnet but later adopted Maxwell's theory of magnetism (1873). 


\section{SWITCH TURNED OFF}

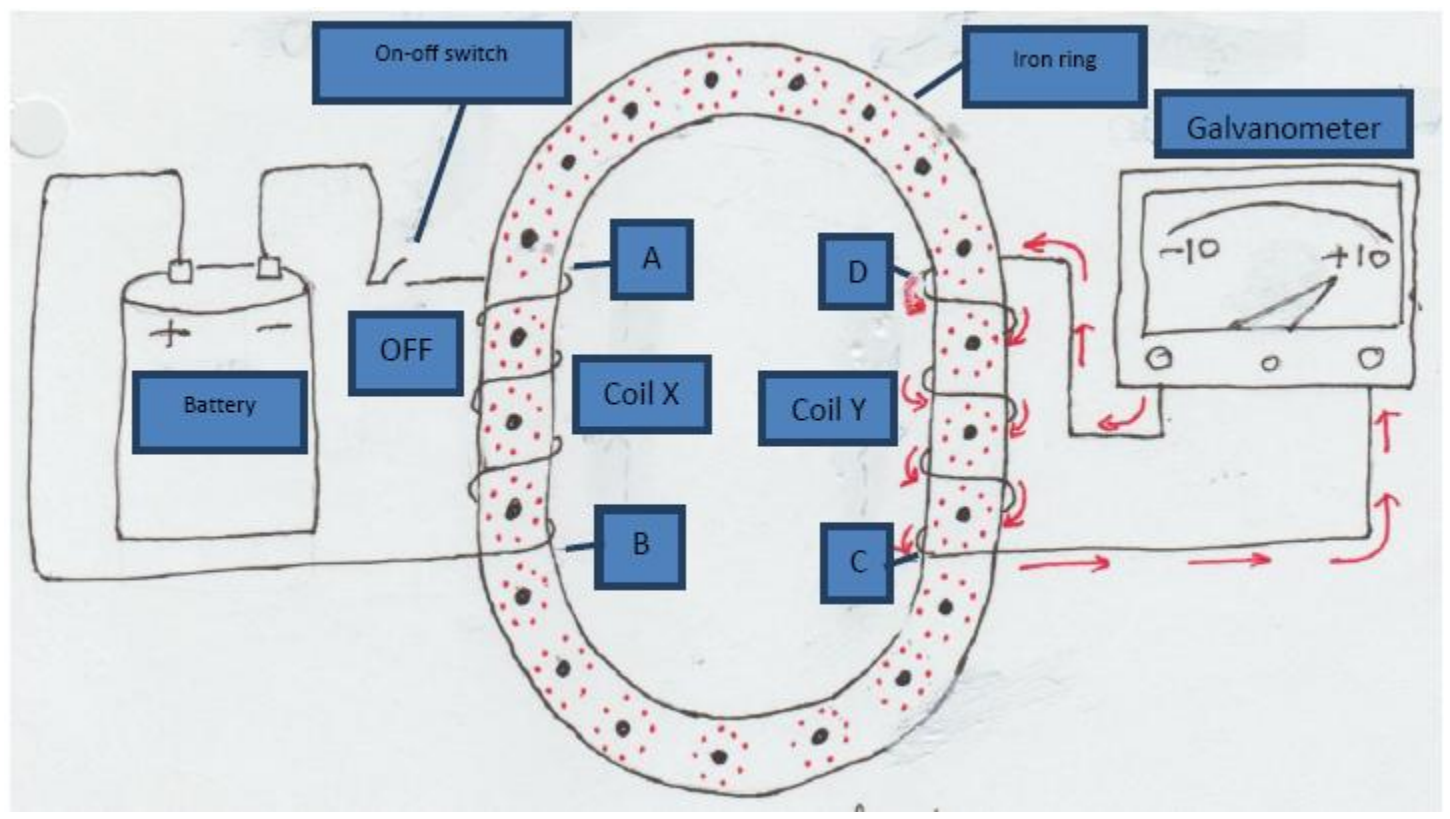

Figure11. switch is turned off

In Figure 11 switch is turned off and we see the previously magnetized atoms in iron ring going back to the neutral position. A reverse centrifugal force due to a reverse torque travels from $D \rightarrow C$ during demagnetization causing a reverse electrical current in coil $Y$ as shown by an opposite deflection in the galvanometer connected to coil Y.

\section{DEMAGNETIZED IRON RING ATOM INSIDE COIL Y WHEN SWITCH IS TURNED OFF}

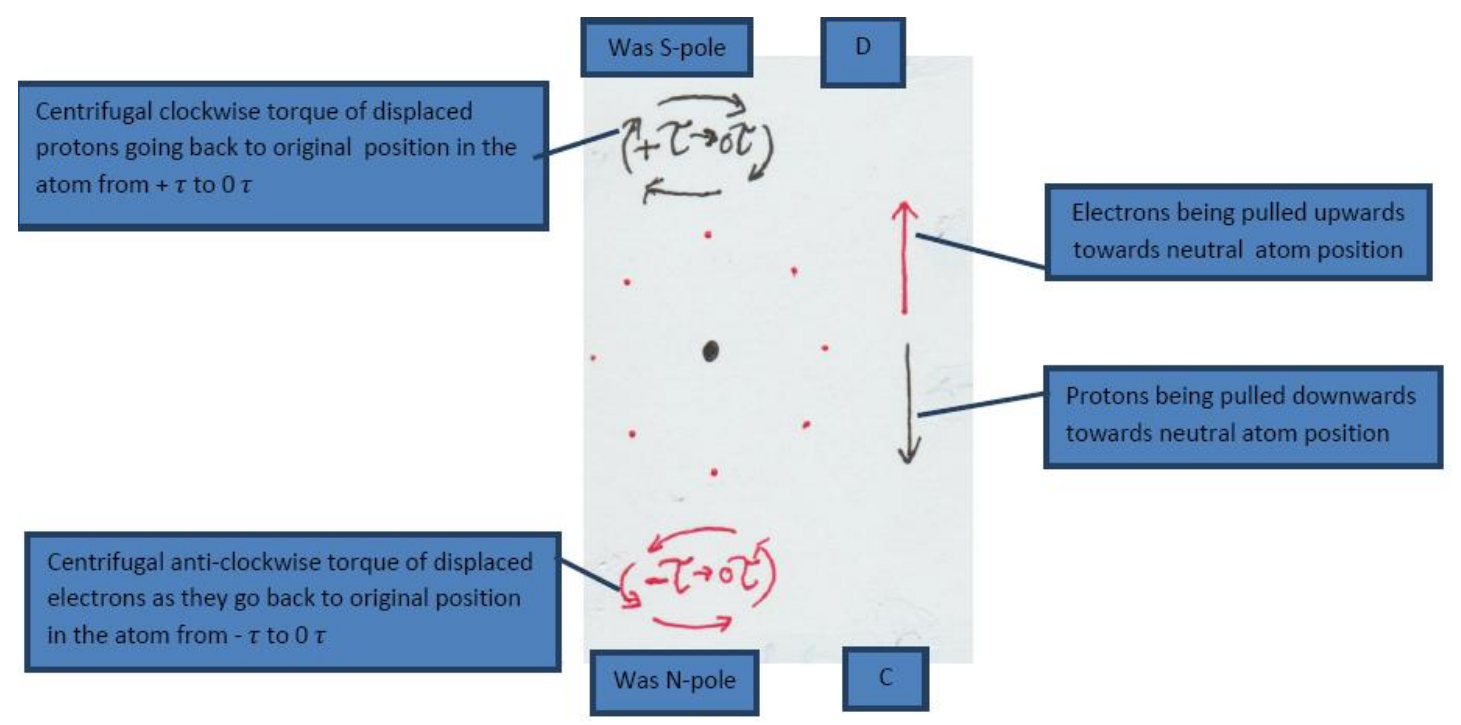

Figures12. Demagnetized atom in iron ring between $C$ and D inside coil $Y$

Fig 12 shows the transient reverse change in configuration of the iron core ring atoms inside coil $Y$ as they go back to a de-magnetized state when the switch is turned off. The inner electrons in the iron ring atoms which were temporarily magnetized by electric current in coil X sequentially from $C \rightarrow D$ now move back around their respective protons sequentially from $D \rightarrow C$. The electrons lose their torque from $-\boldsymbol{\tau} \rightarrow \mathbf{0} \boldsymbol{\tau}$ in a counter-clockwise direction and are pulled upwards and the protons lose their torque from $+\boldsymbol{\tau} \rightarrow \mathbf{0} \boldsymbol{\tau}$ in a clockwise direction and this wave of demagnetization travels from $D \rightarrow C$. During this process of losing their torque and associated return of the inner electrons around their respective protons of the atoms in the iron ring, they exert a transient travelling reverse centrifugal circular force from $D \rightarrow C$ acting on the free valence electrons of coil $Y$ in the opposite direction as compared to when the switch was turned on resulting in_a transient induced electric current and galvanometer deflection in the opposite direction in coil $\mathrm{Y}$ as compared to when the switch was turned on. 


\section{Mathematical Calculations}

The author did an extensive research into the Lorentz force law of 1893 involving the cross product vectors and tried to combine it with Gill's electronic theory of magnetism (1964)

$$
\mathbf{F}=q(\mathbf{E}+\mathbf{v} \times \mathbf{B})
$$

A lot of effort was made to reconcile Gill's electronic theory of magnetism with Lorentz law (1893) but there were two major concerns. Gill's electronic theory of magnetism treats magnetism as a combination of electron dependant and proton dependant forces of a re-configured magnetized atom. Lorentz in 1893 seems to address the magnetic force as a single force B. Also, the author feels that cross products are more algorhythmic and less physics.

Thus, the author decided to develop his own equations to explain the above discussion.

We have seen how the rotating electrons in coil X moving from $A \rightarrow B$ in a tubular manner and the author derived the following dot product vectors with the application of Coulomb's law.

Calculating the Force on a Proton of an Atom in the Centre of Iron Ring from the ELECTRIC COIL

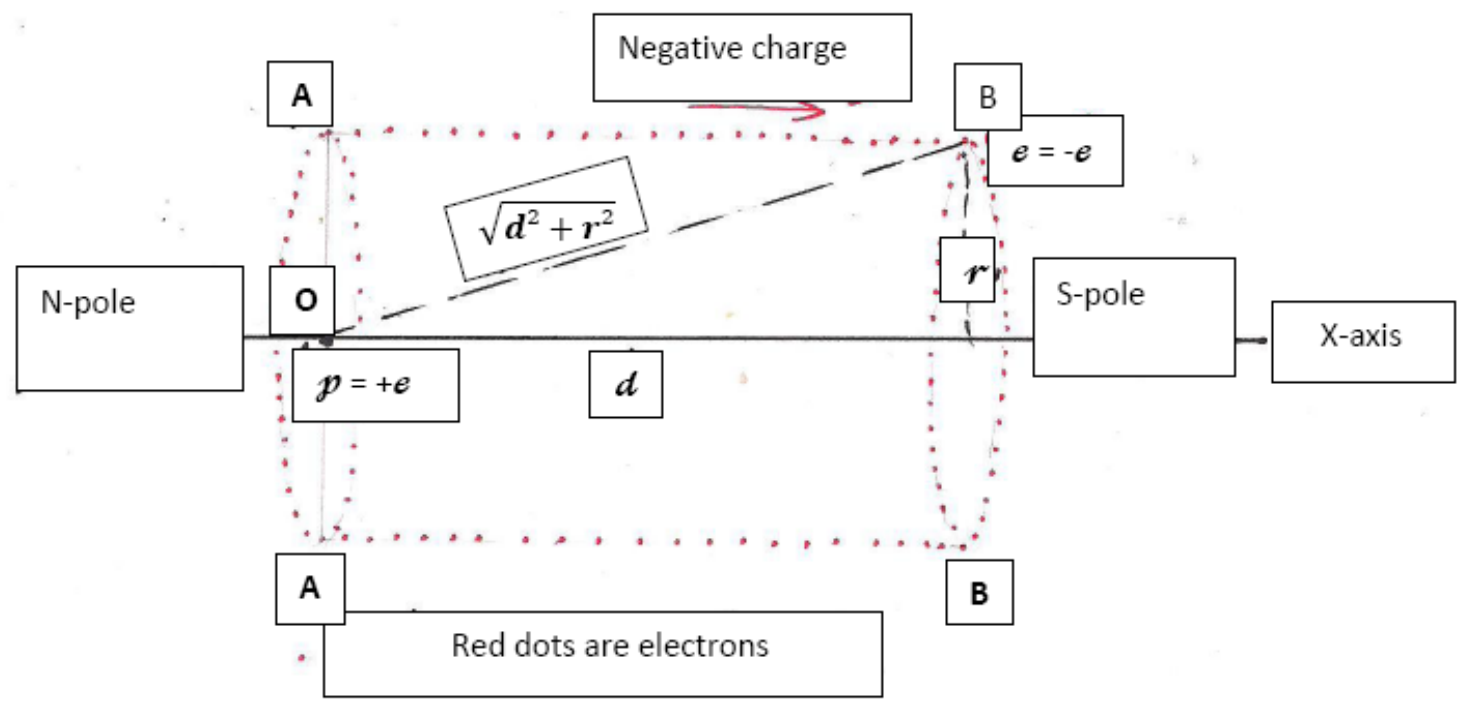

Figure 13

$\mathrm{AA}$ to $\mathrm{BB}$ is a cylindrical conducting coil $\mathrm{X}$ from point $\mathrm{A}$ to point $\mathrm{B}$ around an iron core which is part of the iron ring seen along the $\mathrm{X}$-axis. At the centre $\mathrm{O}$ of the iron ring along the $\mathrm{X}$-axis, we have placed a proton of an atom of the iron ring with a charge $p=+e$. As the electrical current is flowing through the coil, we have electrons flowing from $A \rightarrow B$ and we have an electron with a charge- $e$ at point $\mathrm{B}$ in the conducting coil. The distance $A \rightarrow B=d$ and $r$ is the radius of the coil. Applying Pythagoras theorem, the distance $O B=\sqrt{d^{2}+r^{2}}$. Using Coulomb's law, the force of attraction between $+p=+e$ at 0 and $-e$ at point $\mathrm{B}$ is

$\boldsymbol{F}=\frac{\boldsymbol{k}(+e)(-e)}{\left(\sqrt{\left.\boldsymbol{d}^{2}+r^{2}\right)^{2}}\right.}=-\frac{\boldsymbol{k} e^{2}}{\boldsymbol{d}^{2}+r^{2}}$ (newtons- this will be the force unit in all subsequent calculations)

The minus sign indicating attraction between the negative electron at $B$ and the positive proton of an atom of the iron ring at point 0 . The bold letter equations are vectors.

$\boldsymbol{\theta}$ is the angle between $\mathrm{OB}$ and the $\mathrm{X}$-axis and the vector of $\mathrm{OB}$ along the $\mathrm{X}$-axis is given by the dot product as

$F \cos \theta=-\frac{k e^{2}}{d^{2}+r^{2}} \cos \theta=-\frac{k e^{2}}{\left(d^{2}+r^{2}\right)} \cdot \frac{d}{\sqrt{d^{2}+r^{2}}}$.

When $d$ is very large compared tor, then $r^{2}$ is still smaller and can be ignored and the above vector along $\mathrm{X}$-axis is

$F \cos \theta=-\frac{k e^{2}}{d^{2}} \cdot \frac{d}{\sqrt{d^{2}}}=-\frac{k e^{2}}{d^{2}}$. 
As the electrons move from A to B, $d$ varies from $d=0$ at A to $d=d$ at B and if $d_{\mathrm{x}}$ is an infinitesimally small distance along $\mathrm{A}-\mathrm{B}$, then the vector along the $\mathrm{X}$-axis becomes

$F=\int_{0}^{d}-\frac{k e^{2} d d_{\mathrm{x}}}{d^{2}}=-k e^{2} \int_{0}^{d} \frac{d_{\mathrm{x}}}{d}$

The derivative of $\log d=\frac{1}{d}$, so the anti-derivative of $\frac{1}{d}=\log d$ and integrating from 0 to $d$

$F=-k e^{2}[\log d]_{0}^{d}=-k e^{2}(\log d-\log 0)=-k e^{2} \cdot \log d$ Equation 1a

As the number of electrons rotating in a circular manner in every coil is $\boldsymbol{a}$ at any particular time (where $\boldsymbol{a}$ depends on radius $r$ and the total $\boldsymbol{a}$ will depend on the number of coils at each level and the number of electrons flowing from $A$ to $B$ is $\boldsymbol{b}$ at that particular time, then the total vector from equation 1a along positive $\mathrm{X}$-axis direction from $A \rightarrow B$ is

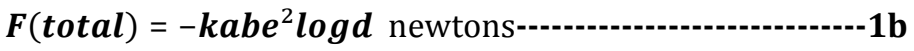

Total flowing charge at any particular time in the coil X is $\frac{a b(-e)}{\boldsymbol{s e c}}=-\boldsymbol{Q} \frac{\text { coulombs }}{\boldsymbol{s e c}}=-\boldsymbol{I}$ amperes . Substituting total charge in motion in the coil X from $A \rightarrow B$ along the X-axis in equation $\mathbf{1 b}$, the dot product force exerted by the flowing electrons on the proton of an atom in iron ring is:

$F($ total $)=\boldsymbol{k}(\boldsymbol{a b}(-\boldsymbol{e})(+\boldsymbol{e}) \cdot \log d=-\boldsymbol{k I e} \cdot \log d$ newtons

If the total number of atoms in iron ring arranged in a linear manner from $A \rightarrow B$ is $\boldsymbol{m}$, and if the number of atoms at each cross section of the iron ring is $\boldsymbol{n}$, then the dot product force acting on the atom at each level along $A \rightarrow B$ is

$\boldsymbol{F}=-\frac{\text { kIe.logd }}{\boldsymbol{m} \boldsymbol{n}}$ newtons................Equation $S$.

As the proton in the above discussion is part of an atom in the iron ring, it undergoes with the help of the dot product force calculated by Equation $\mathrm{S}$, a pull in the direction $A \rightarrow B$.

Also, the total dot product repelling force exerted by the flowing electronsin coil Xfrom $A \rightarrow B$ on the inner electrons of the atoms in the iron ring is:

$F($ total $)=+k(-I)(-e) \log d=+k I e . \log d$ newtons.

As the atoms in the iron ring are in a homogeneous medium, the calculations can be done from the central axis of the iron ring for all the atoms in the iron ring.

If the total number of atoms arranged in a linear manner from $A \rightarrow B$ is $\boldsymbol{m}$, and if the number of atoms at each cross section of the iron ring is $\boldsymbol{n}$, then the dot product force acting on inner electrons of the atom in the iron ring at each level along $A \rightarrow B$ is

$\boldsymbol{F}=+\frac{\boldsymbol{k I e . l o g d}}{\boldsymbol{m} \boldsymbol{n}}$ newtons.................Equation $N$.

$$
F=-\frac{k I e . \log d}{m n} \text { newtons }
$$

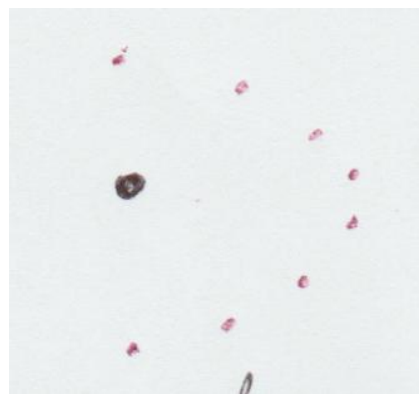

$$
F=+\frac{k I e . \log d}{m n} \text { newtons }
$$

Figure14. Magnetized atom in iron ring

The dot product force from the electrical current in coil X causes $F=+\frac{k l e . l o g d}{m n}$ newtons as in Equation $\mathrm{N}$ which attracts the inner electrons of the atom in the iron ring. 
The dot product force because of electrical current in coil X causes $\boldsymbol{F}=-\frac{\boldsymbol{k I e} \text { logd }}{\boldsymbol{m} \boldsymbol{n}}$ newtons as in Equation $\mathrm{S}$ which attracts the protons of the atom to cause the atom in iron ring to behave like a magnet according to Gill's electronic theory of magnetism.

Torque $\boldsymbol{\tau} \infty \boldsymbol{I}$ and also $\boldsymbol{\tau} \infty$ number of coils at each level from $A \rightarrow B$ which has no role in the forces along the X-axis.

$\boldsymbol{I}$ is electric current in Amperes in the coil, $\boldsymbol{k}$ is the Coulomb's constant, $+\boldsymbol{e}$ is the positive charge of a proton, $-\boldsymbol{e}$ is the negative charge of an electron, $\boldsymbol{d}$ is the longitudinal distance AB of the coil $\mathrm{X}$ in meters. $\boldsymbol{b}$ is the number of electrons flowing along X-axis per second, $\boldsymbol{a}$ is the number of electrons flowing in a circular manner per second causing a centripetal force at each level. $\boldsymbol{a} \boldsymbol{b} \boldsymbol{e}=\boldsymbol{Q}$ or total charge in Coulombs moving along the $\mathrm{X}$-axis in the coil per second.

It should be noted that the atoms in the iron ring are magnetized sequentially from $A \rightarrow B$ along with the negative electron dependant electric current flowing from $A \rightarrow B$ in coil X.

The torque- $\boldsymbol{\tau}$ on the protons and $+\boldsymbol{\tau}$ on the electrons of an atom of the iron ring is caused by the circular rotation of the flowing electrons in coil X perpendicular to the $\mathrm{X}$-axis and has no role in the forces along $\mathrm{X}$ - axis.

\section{RESULT}

It has been shown how a negative centripetal force from the negative electron flow of electric current in coil X travelling from $A \rightarrow B$ causes a change in configuration of the atoms in the iron ring so that they become tiny magnets and this magnetization travels from $A \rightarrow B \rightarrow C \rightarrow D \rightarrow A$ in the iron ring all the way round and as this change in atomic configuration travels from $C \rightarrow D$ in the iron ring, it results in a centrifugal force travelling from $\mathrm{C}$ to $\mathrm{D}$ to cause an electric current in coil $\mathrm{Y}$ which is in opposite direction to the electric current in coil X owing to a 180 degree change in direction. When the electric current in coil $\mathrm{X}$ is switched off, the reverse centrifugal force due to the iron ring atoms assuming their normal configuration as compared to before when the coil X was switched on travels in the iron $\operatorname{ring}$ from $A \rightarrow$ $D \rightarrow C \rightarrow B \rightarrow A$ and we have an electric current in coil Y with an opposite deflection of the galvanometer as compared before.

The torque and the resultant travelling centrifugal force gets manifested all along the iron ring on the surface of the iron ring only at the time of magnetization and an opposite torque will be manifested only at demagnetization as the atoms return to their neutral position. The latter causes an electrical current in coil Y which is opposite to that seen at magnetization and is seen as an opposite galvanometer deflection as compared to before. A magnet would otherwise manifest its negative and positive forces at its two ends only.

\section{North Magnetic Pole is the Electron Dependant Negative Magnetic Pole}

In 1826, Andre -Marie Ampere talked of the twisting of the electrons during magnetization and application of his right hand grip rule along with Gill's electronic theory of magnetism (1964) helps to show that the north magnetic pole is the exposed inner electron dependant negative magnetic pole.

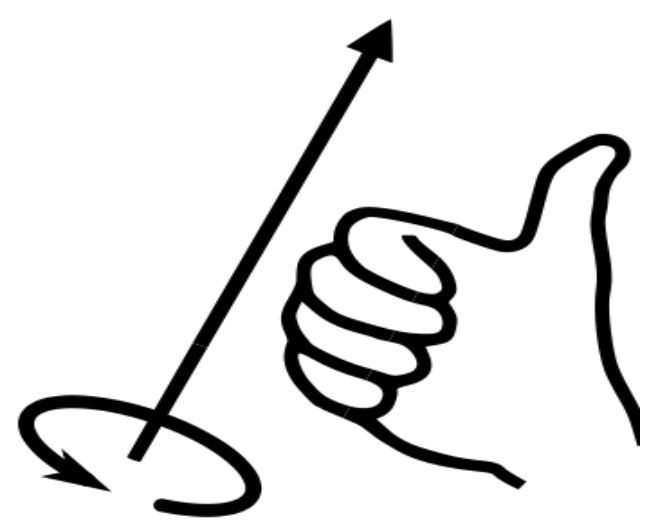

Figure15

According to Amperes right hand grip rule(1826), if the fingers of the right hand are curled around the coil in the direction of current flow (conventional current is flow of positive charge) and the thumb points in the overall direction of the positive current flow, then the thumb points in the direction of the north magnetic pole. According to Gill's electronic theory of magnetism(1964) and as discussed above, the 
direction of the thumb points to extra inner electrons of the iron core atom subjected to a pull in the direction of the thumb and a torque $(\boldsymbol{\tau})$ in the direction of the curled fingers owing to the centripetal force of the electric current in the conducting coil. Thus, the north magnetic pole is the same as the exposed inner electron dependant negative magnetic pole.

Corollary (1) Gill's modified right hand grip rule for a magnet- If the fingers of the right hand are curled around a magnet and the thumb is pointed in the direction of the north /negative magnetic pole of the magnet, then the non-moving inner electrons at the north magnetic pole will be torqued $(\boldsymbol{\tau})$ in the direction of the curl of the fingers.

Ampere talked of a force on the surface of a magnet but he later accepted Maxwell's theory of magnetism .

\section{Asymmetry Issue Resolved with the Application of Gill's Electronic Theory of MAGNETISM (1964)}

This issue was raised by A. Einstein in his 1905 paper on Special Relativity and by Richard P. Feynman in 1941. The issue arose because of Maxwell's dipole theory of magnetism (1873) which treats the magnetic force as a single force.

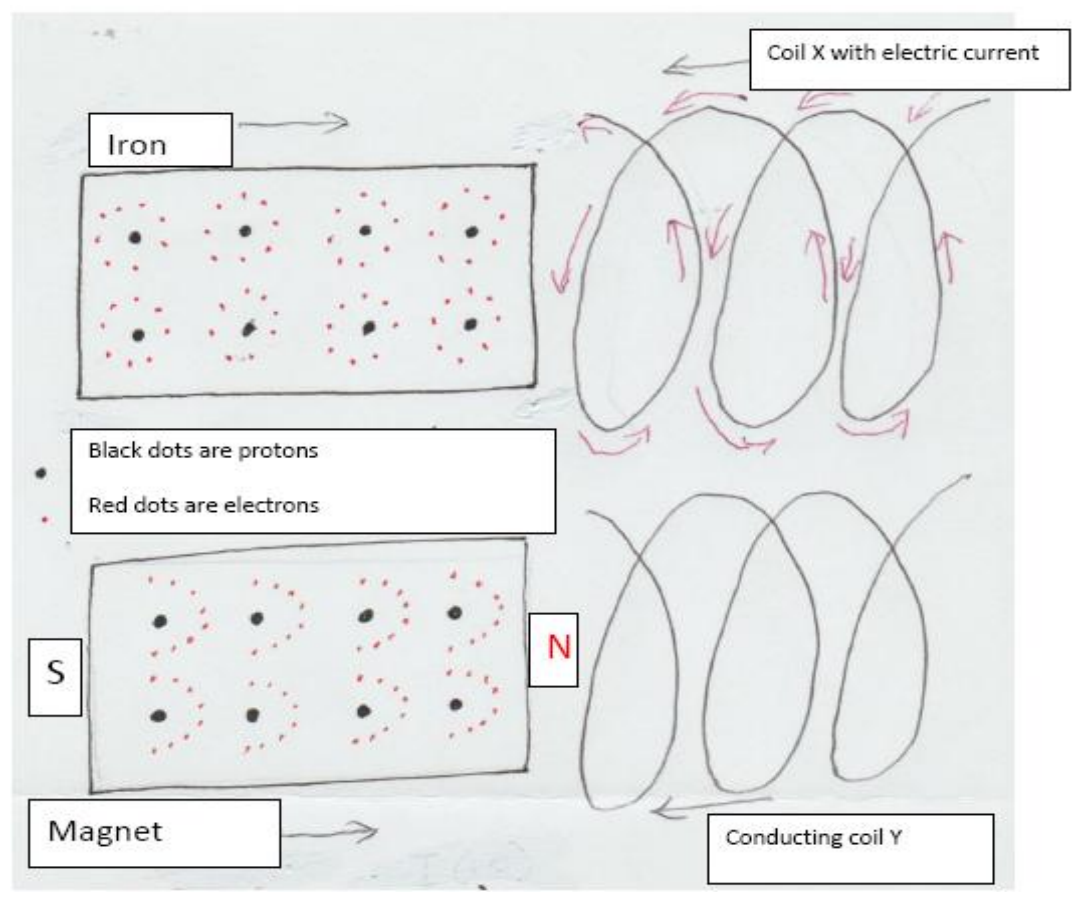

Figure 16

In the upper part of above figure, we have an electric coil X moving towards a piece of iron. According to Gill's electronic theory of magnetism, the piece of iron will get magnetized and there will be a changing magnetic behavior so long as there is change in relative motion between the electric coil and the iron piece which gets magnetized. This is similar to the coil X experiment in Faraday's iron ring experiment.

In the lower part of above figure, we have a magnet NS moving towards and into the coil Y. The torqued North magnetic pole and the oppositely torqued South magnetic pole along with the movement of the magnet towards and into the coil will cause a flow of the free valence electrons in coil Y as an electric current. This electric current will continue so long as there is relative movement between the magnet and coil Y. This is similar to the coil Y situation in the Faraday's iron ring experiment.

The asymmetry flaw was in the acceptance of Maxwell's dipole theory of magnetism (1873) and the consequent Maxwell's electrodynamics. Also, dealing with the magnetic force as a single force and not treating the magnetic force as a combination of two forces from the proton dependant south magnetic pole and the electron dependant north magnetic pole of a magnet was incorrect.

A. Einstein's June 1905 article 'On the Electrodynamics of Moving Bodies' makes interesting reading as he tries to sort out the asymmetry issue which never existed if you apply Gill's electronic theory of magnetism(1964. The fault was in his accepting magnetic force as a single force put forward by Maxwell in 1873 and what an exercise over something called asymmetry which never existed in the first place as explained above. 


\section{Magnetization And More Calculations}

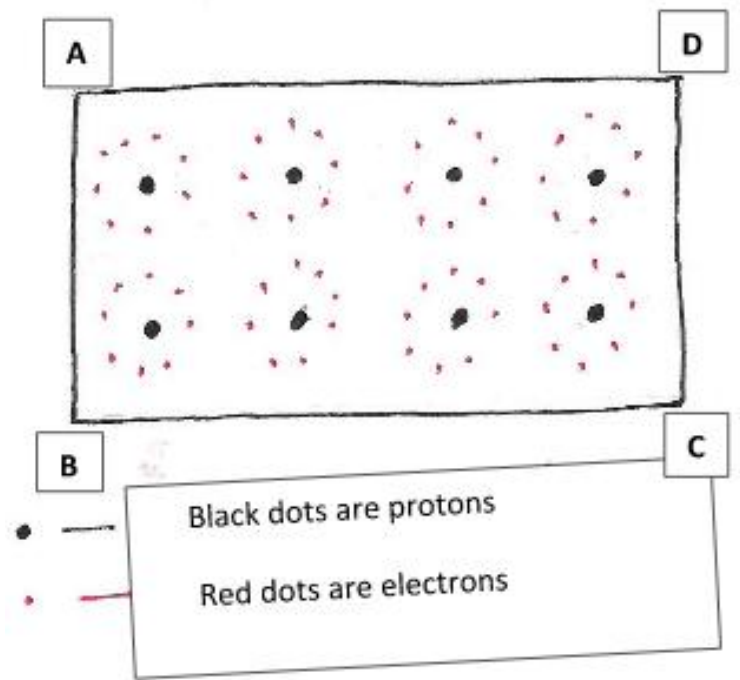

Figure 17

Figure 17 above shows a piece of iron ABCD which is showing atoms with central protons each surrounded by electrons in a neutral state.

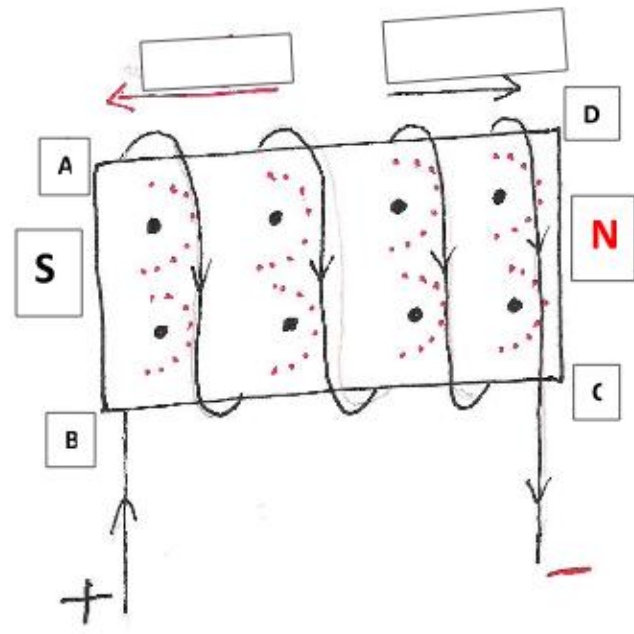

Figure 18

In figure 18, the piece of iron ABCD is placed in an electrical coil which has an electric current $+I(+e)$ moving from + to -according to the electric current nomenclature. We could also state that we have an electric current $-I(-e)$ flowing along with the valence electrons in the opposite direction in the electric coil. As discussed before, the movement of free valence electrons in the electric coil will repel some of the inner electrons of each atom in the figure $A B C D$ towards $C D$ which will develop a temporary non-moving negative charge and behave as the magnetic N-pole according to Ampere's right hand rule (1826). As some of the inner negative charges or electrons in the figure $A B C D$ have been pushed towards $C D$, the $A B$ end is left with an exposed non-moving positive charge and will behave as the magnetic $S$-pole of the temporary magnet $A B C D$. If the length of the above iron bar is ' $d$ ' and the number of atoms arranged linearly from $A B$ to $C D$ is ' $m$ ' and the number of atoms in a cross-section is ' $n$ ', then applying the formulae derived above:

On surface $A B, \boldsymbol{F}=-\frac{\boldsymbol{k I e}(\log d)^{2}}{m \boldsymbol{n}} \cdot \boldsymbol{n}=-\frac{\boldsymbol{k I e}(\log d)^{2}}{m}$ newtons.................Equation $S$.

On surface CD, $\boldsymbol{F}=+\frac{\boldsymbol{k I e}(\boldsymbol{l o g} d)^{2}}{\boldsymbol{m} \boldsymbol{n}} \cdot \boldsymbol{n}=+\frac{\boldsymbol{k I e}(\log d)^{2}}{\boldsymbol{m}}$ newtons................Equation $N$.

In the steady state of magnetization, we have the forces manifesting only from the N-pole and the S-pole of the magnet. 


\section{Calculating the Tesla unit}

The force of attraction between- $\boldsymbol{Q}$ at $\mathbf{N}$-pole and $+\boldsymbol{Q}$ at $\mathbf{S}$-pole

$F=\frac{k(-Q)(+Q)}{d^{2}}$ newtons $=-\frac{k Q^{2}}{d^{2}}$ newtons.

$\boldsymbol{d}$ is the distance between the two magnetic poles in meters and $\boldsymbol{k}$ is Coulomb's constant.

The N-pole has an exposed negative charge $-\boldsymbol{Q}=\boldsymbol{n}(-\boldsymbol{e}) \operatorname{coulom} \boldsymbol{b s}$ where $\boldsymbol{n}$ is the number of exposed inner electrons at magnetization.

The S-pole has an exposed positive magnetic charge $+\boldsymbol{Q}=\boldsymbol{n}(+\boldsymbol{e}) \boldsymbol{c o u l o m} \boldsymbol{b s}$ where $\boldsymbol{n}$ is the number of exposed protons at the $\mathrm{S}$-pole.Then, the above equation becomes

$F=\frac{k(-n e)(+n e)}{d^{2}}$ newtons $=-\frac{k n^{2} e^{2}}{d^{2}}$ newtons or

$n^{2}=-\frac{F d^{2}}{k e^{2}}$ or in units it is $\frac{N m^{2}}{\left(N m^{2} C^{-2}\right) C^{2}}$.

If $\boldsymbol{F}=-\mathbf{1}$ newton and $\boldsymbol{d}=\mathbf{1}$ meter, then we have

$n^{2}=\frac{1}{k e^{2}}$ or

$n=\frac{1}{\sqrt{k} \cdot e}=(\sqrt{k} \cdot e)^{-1}$.

$1 e \approx 1.6 \times 10^{-19} \mathrm{C} ;$ Coulombs constant $k=8.99 \times 10^{9} \mathrm{Nm}^{2} \mathrm{C}^{-2}$.Applying these, we get

$n=\frac{1}{\sqrt{k} \cdot e}=\frac{1}{\sqrt{\left(8.99 \times 10^{9}\right)} \cdot\left(1.6 \times 10^{-19}\right)}=\frac{1}{(94815.61)\left(1.6 \times 10^{-19}\right)}=\frac{1}{1.52 \times 10^{-14}}=6.6 \times 10^{13}$.

Tesla: If we have a magnetic north pole with $6.6 \times 10^{13}$ exposed inner electrons and at one meter, we have a south magnetic pole with $6.6 \times 10^{13}$ exposed protons, then the magnetic force of attraction between the two magnetic poles is one Tesla.

\section{DisCUSSION}

Faraday's iron ring experiment (1831) is explained by applying Gill's electronic theory of magnetism (1964) and dot product formulae are offered preceded by their derivation by the author. The above explanation also shows that we are dealing with a single phenomenon which is the interaction between forces emanating from the electrons and the protons of an atom, whether it is electricity generation or whether it is what we perceive as a magnetic force which is a combination of the electron dependant negative force and the proton dependant positive force.

Dot product equations derived with the application of Gill's electronic theory of magnetism (1964) should be used as they are more scientific and will lead to better applications.

Gill's electronic theory of magnetism (1964) points out that there are no pre-existing dipoles in a ferro-magnet and shows how otherwise neutral atoms in the iron ring undergo a change in configuration when magnetized to behave like tiny magnets. When demagnetized, these atoms resume their neutral configuration. It was shown in the above discussion that this magnetization at the atomic level in the iron ring travels in the direction of the negative electric current flow from the magnetic north pole towards the magnetic south pole and continues all the way around as each atom is magnetized. Further, it was explained that a centrifugal force develops owing to reconfiguration of the iron atoms into tiny magnets which travels all the way around in the iron ring as each atom causes a similar change in the next atom and the chain continues. This travelling centrifugal force from the iron ring manifests only at the time of magnetization and a reverse travelling centrifugal force will manifest only at the time of demagnetization and there is no travelling centrifugal force once the magnetization is in the steady state. In a steady state of magnetization, only the free ends of a magnet will display the torqued electron based negative or north magnetic pole and the oppositely torqued proton dependant positive or south magnetic pole.

\section{ConCLUSION}

Gill's electronic theory of magnetism (1964) helps in explaining the 1831 iron ring experiment of Michael Faraday and offers a dot product based calculation unlike Maxwell's magnetic dipole theory (1873) which forces us to indulge in cross products.

The calculation of the Tesla unit is also submitted. 
Combining Ampere's circuit law (1826) with Gill's electronic theory of magnetism (1964) helps in showing that the electron dependant negative magnetic pole is the magnetic $\mathrm{N}$-pole and the proton dependant positive magnetic pole is the magnetic S-pole.

Electromotive forces and the magnetic forces are because of one phenomenon only which is the interaction of proton based positive and electron based negative forces manifesting as magnetized atoms in the iron ring due to the electron based electric current in coil X.

This article resolves the asymmetry issue raised by A. Einstein at the beginning of his 1905 article on Special Relativity and by Richard Feynman in 1943. Applying Gill's electronic theory of magnetism shows that there has never been any asymmetry in the first place.

In the end, I am grateful to a physicist Professor Amitabha Mukherjee, former head of department of physics at Delhi University, New Delhi, India for sparing time from his very busy schedule to go over this article with me.

\section{REFERENCES AND NOTES}

1. Coulomb's law by Charles Augustin de Coulomb (1784))

2. A Treatise on Electricity and Magnetism by James Clerk Maxwell

3. Ampère's circuital law by Andre Marie Ampere (1823)

4. Bohr model by Niels Bohr and Ernest Rutherford (1913)

5. Lorentz force by Hendrik Lorentz (1893)

6. On the electrodynamics of moving bodies By A. EINSTEIN June 1905

7. Tesla unit by Nikola Tesla

8. Elementary charge units

Citation: Avtar Singh Gill. "Faraday's Iron Ring Experiment (1831) Explained with Gill's Electronic Theory of Magnetism (1964)", American Research Journal of Physics, vol 3, no. 1, 2017, pp. 1-17.

Copyright (c) 2017 Avtar Singh Gill, This is an open access article distributed under the Creative Commons Attribution License, which permits unrestricted use, distribution, and reproduction in any medium, provided the original work is properly cited. 